\title{
Approach to Quantitative Detection of CD146 with the Label- free Protein Biosensor Based on Imaging Ellipsometry
}

\author{
Yu Niu ${ }^{a, b}$, Li Liu $^{a, b}$, Xiyun Yanc, Gang Jin ${ }^{a, *}$ \\ ${ }^{a}$ NML, Institution of Mechanics, Chinese Academy of Sciences, \#15, Bei-si-huan West \\ Road, Beijing 100190, China; \\ ${ }^{\mathrm{b}}$ Graduate University of the Chinese Academy of Sciences, \#19, Yu-quan Road, Beijing \\ 100049, China; \\ ${ }^{c}$ Institution of Biophysics, Chinese Academy of Sciences, \#15, Da-tun Road, Beijing \\ 100101, China; \\ *Corresponding author. Tel./fax: +86 10 82544138. E-mail address: gajin@imech.ac.cn.
}

\begin{abstract}
CD146 glycoprotein belonging to cell adhesion molecules is considered to be a novel target on endothelial cell involved in tumor angiogenesis. The biosensor based on imaging ellipsometry (BIE) which is performed in null and off-null mode is used for CD146 detection as a trial by the following steps. Firstly, anti-CD146 antibody as ligand is immobilized on Protein G modified silicon substrate. Then, CD146 test is carried out and its calibration curve is established for the requirement of quantitative detection. Finally, 18 serum samples are detected quantitatively and their results are validated by ELISA's. The sensitivity for CD146 detection achieves the order of $\mathrm{ng} / \mathrm{ml}$ and the relationship between BIE signal y (grayscale value) and CD146 concentration $x(\mathrm{ng} / \mathrm{ml})$ is $y=3.3 \ln (x)+91$.3. Compared with ELISA's, the majority of results are in agreement, and the results of two approaches have significant statistic relevance.
\end{abstract}

Keywords: CD146 detection, biosensor, imaging ellipsometry.

\section{INTRODUCTION}

As cancer becomes one of the most severe dangers for human beings [1], a lot more attention has been paid to the field of cancers detection. So far, it has been proved that detection of tumor markers is an efficient method to make a prompt and early-stage diagnosis [2]. Therefore, the need for novel and effective methods which can detect tumor markers rapidly, sensitively and reliably is consequently being subjected to extensive interest.

The concept of biosensor based on imaging ellipsometry for visualization of biomolecular interactions was reported in 1995 [3, 4]. Imaging ellipsometry with the advantages of ellipsometry and microscopy simultaneously could measure the surface concentration of protein adsorbing layer accurately without any labeling. It provides possibility to apply BIE's function in a broad range of various biological systems, especially for the detection of protein interactions [5,6]. So far, several bio-targets, such as five markers of Hepatitis B [7], Phage M13KO7 [8], and SARS Virus [9], have been successfully tested with the biosensor.

CD146 is a member of cell adhesion molecule (CAMs) [10,11]. In pathology, it is originally identified as a biomarker for melanoma detection [12]. A former study indicates that CD146 may play an important role in promoting melanoma progression. Enforced expression of CD146 on melanoma cells increases tumor growth, and decreasing CD146 expression releases tumor formation [13]. Nowadays, CD146 is considered as a novel target on endothelial cell involved in tumor angiogenesis [10].

Sensors, Cameras, and Systems for Industrial, Scientific, and Consumer Applications XII,

edited by Ralf Widenhorn, Valérie Nguyen, Proc. of SPIE-IS\&T Electronic Imaging, SPIE Vol. 7875

78750R - @ 2011 SPIE-IS\&T · CCC code: 0277-786X/11/\$18 - doi: 10.1117/12.871972

Proc. of SPIE-IS\&T/ Vol. 7875 78750R-1 
In this investigation, CD146 is chosen to be a model of tumor markers and then tried to detect quantitatively with BIE.

\section{MATERIALS AND METHODS}

\subsection{BIE and its detection principle}

To perform molecular interaction as the high throughput immunoassays, BIE has been developed in our laboratory $[5,6,14]$. Now, it is mainly composed of a micro-fluidic reactor array system and an imaging ellipsometry reader.

A micro-fluidic reactor array system including 48 independent flow channels is used to fabricate protein microarray for high throughput detection by a series of continuous processes, including surface patterning, solution delivery, ligand immobilization, blocking, target capture, rinsing, etc [5,6]. The micro-fluidic array system is composed of four main parts: sample reservoir, multi-cell array, multi-channels and pumps. When a silicon wafer substrate is placed on the multi-cell array to form closed paths, a patterned microarray can be fabricated because each cell has an independent inlet and outlet for solution delivery. The inlet microchannels are connected to the sample reservoir and the outlet micro-channels are connected with pumps offering negative pressure. Using the micro-fluidic reactor system, various ligands are delivered to different cells to form a sensing array, and each sensing surface can be prepared homogeneously.

Imaging ellipsometry acts as the data acquisition of the microarray. Imaging ellipsometry which combines the power of ellipsometry with microscopy and works in the off-null mode $[3,4]$ is an enhancement of standard single-beam ellipsometry. Upon the high sensitivity requirement for weak affinity biomolecular interactions, its sensitivity has been improved by the polarization setting optimization, the wavelength optimization and an imaging device with low noise. It can be utilized for the visualization of ultra-thin films and slight surface concentration change. Here, it is used to quantify the thickness distribution of protein layers [6, 15]. A slight variation of thickness can be remarkably distinguished by imaging ellipsometry and the result is represented by images in grayscale format ( 8 bits, 0-117 255 grayscale or 10 bits, 1024 grayscale). The grayscale value is approximately linearly proportional to the thickness corresponding to the surface concentration [15].

\subsection{Chemicals, samples and substrates}

Silicon wafers are purchased from Beijing GRINM semiconductor Materials Company. 1-(3Dimethylaminopropyl)-3-ethylcarbodiimide hydrochloride (EDC), succinic anhydride and aminopropyltriethoxysilane (APTES) are purchased from Acros Organics. N-Hydroxy-succinimide (NHS), blocking buffer, PBST buffer (PBS containing $0.05 \%$ Tween-20), Protein $G$ and bovine serum albumin (BSA) are purchased from Sigma-Aldrich. Deionized water is produced by ion exchange demineralization, followed by passing through a Milli-Q plus system from Millipore. Mouse monoclonal antibody to CD146 and all human serum samples are kindly provided by Professor Yan's Group in the institute of Biophysics of Chinese Academy of Sciences.

\subsection{Silicon wafer preparation and surface modification}

Silicon wafer is used as solid surface for imaging ellipsometry biosensor. Due to the need of 48 throughputs, the silicon slides are cut into $25 \times 13 \mathrm{~mm}^{2}$ rectangular pieces. Then, aiming at washing out the organic and inorganic pollution, silicon wafers are cleaned with a mixture of $30 \% \mathrm{H}_{2} \mathrm{O}_{2}$ and concentrated $\mathrm{H}_{2} \mathrm{SO} 4(1: 3 \mathrm{v} / \mathrm{v})$ for 30 minutes. After being thoroughly rinsing with deionized water and pure ethanol, these silicon wafers are treated with an ethanol solution of APTES (5\% APTES and $95 \%$ pure ethanol) and incubated 2 hours at room temperature [16]. Following by intensively rinsing in pure ethanol, the silicon wafers silanized with APTES are reacted with over-saturated succinic anhydride in ethanol for at least 3 hours [17]. After being rinsed with pure ethanol, the silicon wafers are stored in pure ethanol for ligands immobilization. 


\subsection{Detection of CD146}

After surface modification, silicon wafer is placed into the micro-fluidic reactor system to immobilize antiCD146 antibody. Because the anti-CD146 antibody is in ascites, it could not be assembled directly on the surface just depending on covalent immobilization. Therefore, protein $G$ which is a common used method in protein purification is tried to capture anti-CD146 antibody to form sensing layer by the interaction between Fc part of antibody and protein G. Firstly, $10 \mu \mathrm{l}$ of a mixture solution which is prepared with NHS and EDC at the concentration of $0.05 \mathrm{~mol} / \mathrm{ml}$ and $0.2 \mathrm{~mol} / \mathrm{ml}$ in deionized water is added to the micro-fluidic reactor system and passed through the surface at a flow rate of $5 \mu / / m i n$. With NHS and EDC, carboxyl groups on the substrate could react with the amino groups of the protein. Then, $10 \mu \mathrm{l}$ of Protein $\mathrm{G}$ at $0.25 \mathrm{mg} / \mathrm{ml}$ is adsorbed on the substrate at a flow rate of $1 \mu \mathrm{l} / \mathrm{min}$. After that, 20 $\mu$ of anti-CD146 antibody diluted 200 times in PBST buffer as ligands is immobilized specifically to protein $\mathrm{G}$ at a flow rate of $1 \mu \mathrm{l} / \mathrm{min}$. Finally, the silicon surface is blocked by blocking buffer in order to lessen the impact caused by non-specific adsorption of human serum. After these four steps, the sensing surface for CD146 detection is ready in use. When the detection begins, $20 \mu$ of CD146 standard sample or human serum dilution solution is delivered into individual channel to CD146 sensing surface by the micro-fluidic system at a flow rate of $1 \mu \mathrm{l} / \mathrm{min}$. After being rinsed with plenty of PBST and dried under nitrogen, the microarray is sampled and recorded as images in grayscale by the imaging ellipsometry reader.

\subsection{Calibration curve for CD146 quantitative detection}

Calibration curve is used to achieve the goal of quantitative detection. The CD146 standard serum sample is diluted to 3, 9, 27, 81 and 243 times with PBST in term of the equal proportion dilution rule, that is to say, the concentration of CD146 is from $81 \mathrm{ng} / \mathrm{ml}$ to $1 \mathrm{ng} / \mathrm{ml}$. The calibration curve is established three times for verifying the results, and each concentration is measured twice as well. Strictly following the procedures above, results in grayscale of 5 diluted samples and the original sample are obtained and then plotted according to CD146 concentration to establish calibration curve.

\subsection{Quantitative detection of human serum samples}

18 human serum samples are detected by the same procedure to setup calibration curve. In order to ensure CD146 concentration within the range of calibration curve, all serum samples are diluted to 5 times before detection. Each serum is measured twice, and then the results are calculated by the calibration. The BIE results are compared with those from ELISA assay by the correlation analysis.

\section{MATERIALS AND METHODS}

\subsection{Constitution of CD146 sensing layer}

Because Protein G could bind the Fc part of antibody strongly, it is used to immobilize anti-CD146 antibody and then form CD146 sensing surface. By this means, anti-CD146 antibody in complex sample could be purified; meanwhile, oriented immobilization of anti-CD146 antibody is realized on silicon surface. The result for CD146 sensing surface constitution is showed in Figure1. Protein G is covalently adsorbed in all the 6 units of the microarray, and then they are reacted with anti-CD146 antibody in row b and row c units. After that, blocking buffer is added into row c units. Compared with Protein G, the grayscale value of units in the microarray where anti-CD146 antibody is reacted with Protein G increases remarkably from 78.0 to 92.8 . It indicates that anti-CD146 antibody is captured by Protein $\mathrm{G}$ and fabricate sensing layer. 


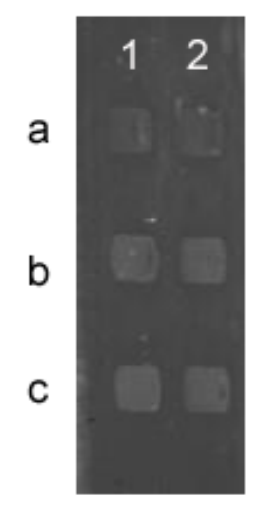

A

\begin{tabular}{|c|c|c|c|}
\hline & Repeat 1 & Repeat 2 & Average \\
\hline a & 77.2 & 78.8 & 78.0 \\
\hline b & 92.3 & 93.5 & 92.8 \\
\hline c & 93.9 & 92.9 & 93.4 \\
\hline
\end{tabular}

B

Figure1: Constitution of CD146 sensing layer. (A) The image in grayscale; (B) Average grayscale value in of image A. Protein G is covalently immobilized in all the 6 units. Then, anti-CD146 antibody is reacted in row $b$ and row $c$ units. After that, blocking buffer is added in to row $c$ units.

\subsection{Detection of CD146}

To detect CD146, blank control and negative control are set to ensure detection accuracy and avoid false positive. Results are demonstrated in Figure $2 \mathrm{~A}$ and the corresponding numerical values are listed in Figure 2B. Protein $\mathrm{G}$ is immobilized in all the 8 units. Then BSA is reacted in row a and row b, while CD146 is added in row $\mathrm{c}$ and row $\mathrm{d}$. After blocked by blocking buffer, CD 146 standard sample is delivered in row $\mathrm{b}$ and row $\mathrm{d}$, while PBST is added in row a and row c. In that case, row a and row b is blank control, whereas, row $c$ is negative control. In the blank control units, compared to row a, grayscale value in row $b$ increases from 89.3 to 90.8 after reacted with standard sample. It is suggested that the non-specific adsorption and other errors may cause an increase of 1.5 grayscale value. In other word, grayscale value increasing beyond 1.5 can be contributed to the specific binding between CD146 and its corresponding antibody. The average grayscale value of sample units is 103.6 while the negative control is 93.2. As a result, it can be concluded that CD146 is captured by anti-CD146 antibody on biosensor surface because the increase is 11.4 which are far more than 1.5 .

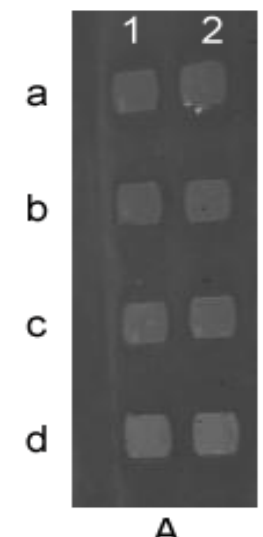

\begin{tabular}{|c|c|c|c|}
\hline & Repeat 1 & Repeat 2 & Average \\
\hline a & 89.1 & 89.5 & 89.3 \\
\hline b & 90.4 & 91.2 & 90.8 \\
\hline c & 93.6 & 92.7 & 93.2 \\
\hline d & 103.2 & 103.9 & 103.6 \\
\hline
\end{tabular}

B

Figure 2 Detection of CD146. (A) The image in grayscale; (B) Average grayscale value in of image A. Protein $G$ is immobilized in all the 8 units. Then BSA is reacted in row a and row b, while CD146 is bound in row $c$ and row $d$. After blocked by blocking buffer, CD 146 standard sample is added in row b and row d, while PBST is added in row a and row c. In that case, row a and row b is blank control, whereas, row $\mathrm{c}$ is negative control. 


\subsection{Calibration for CD146 quantitative detection}

CD146 standard sample is diluted at geometric proportion to 5 grads that are $81 \mathrm{ng} / \mathrm{ml}, 27 \mathrm{ng} / \mathrm{ml}, 9 \mathrm{ng} / \mathrm{ml}$, $3 \mathrm{ng} / \mathrm{ml}$ and $1 \mathrm{ng} / \mathrm{ml}$, respectively, and then reacted with anti-CD146 antibody sensing surface. The calibration curve is showed in Figure 3. It indicates that with the decay of concentration of CD146, the relevant grayscale value declines, and the variable regulation between them appears a logarithm formulation. After a logarithmic transformation of CD146 concentration, the result in grayscale is plotted toward logarithm of CD146 concentration. The 6 points are almost in line of which the slope and $\mathrm{R}^{2}$ (the percentage of total variation in the response that can be explained by factors in regression analysis and the higher R2, the better fit.) are 3.3 and 0.987 , respectively. When CD146 concentration is at $1 \mathrm{ng} / \mathrm{ml}$ and $3 \mathrm{ng} / \mathrm{ml}$, respectively, the biosensor signal shows a significant difference. It can be confirmed that the sensitivity of the biosensor for CD146 detection reaches the order of $\mathrm{ng} / \mathrm{ml}$. The calibration curve appears a logarithm relationship between the biosensor signal $y$ in grayscale and CD146 concentration $x$ in $\mathrm{ng} / \mathrm{ml}$ : $y=3.3 \ln (x)+91.3$. It can be foreseen that the concentration of CD146 between $1 \mathrm{ng} / \mathrm{ml}$ and $243 \mathrm{ng} / \mathrm{ml}$ can be quantitatively detected by BIE with the calibration curve above.

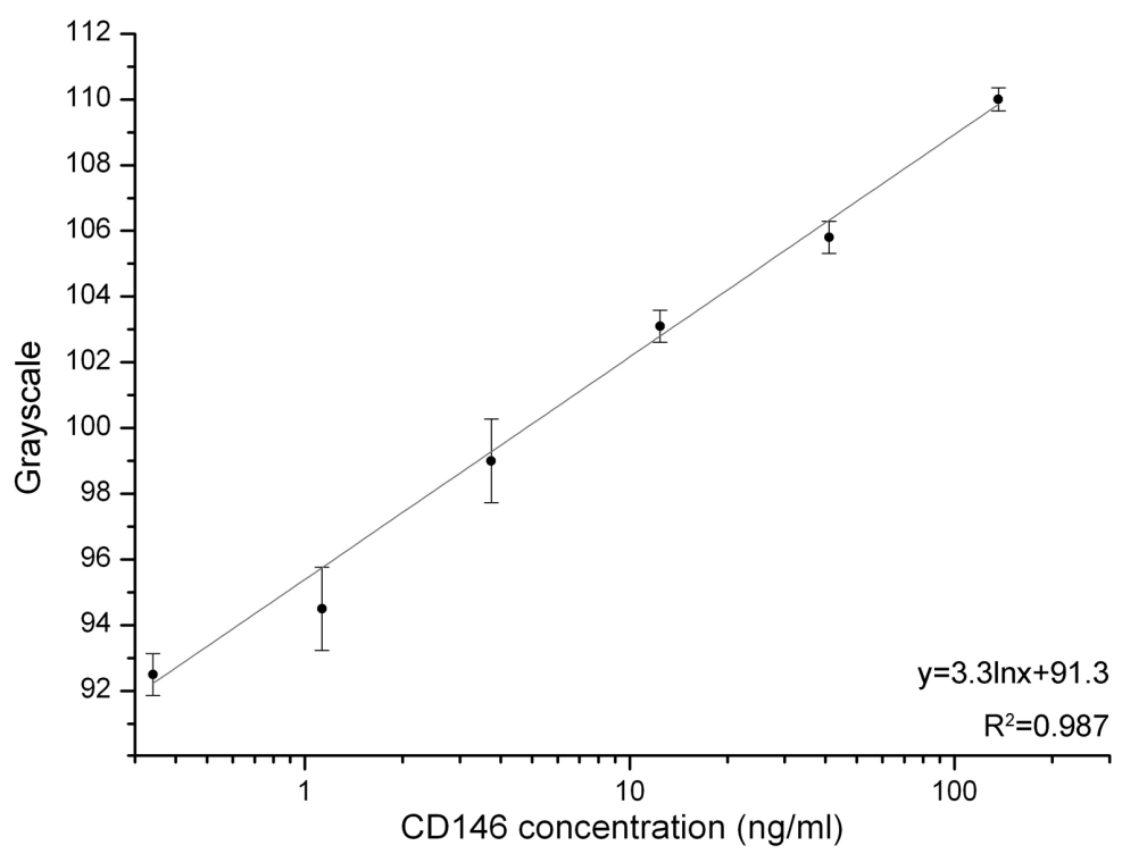

Figure 3 The calibration curve for quantitative detection of CD146.

\subsection{Serum sample detection}

18 human serum samples are tested as a trial to clinical detection. The results of 18 sera are collected and dealt in the same way, and then the results in grayscale can be obtained through home-made software. ELISA, as a conventional biological manner, is set for the comparison. Both tests are performed without knowledge of the clinical status of the patients or the results of another. The difference between ELISA and the biosensor is evaluated with correlation analysis. The index is 0.923 which demonstrates that the result of the two test manner has significant statistic relevance at the level of 0.01 . 


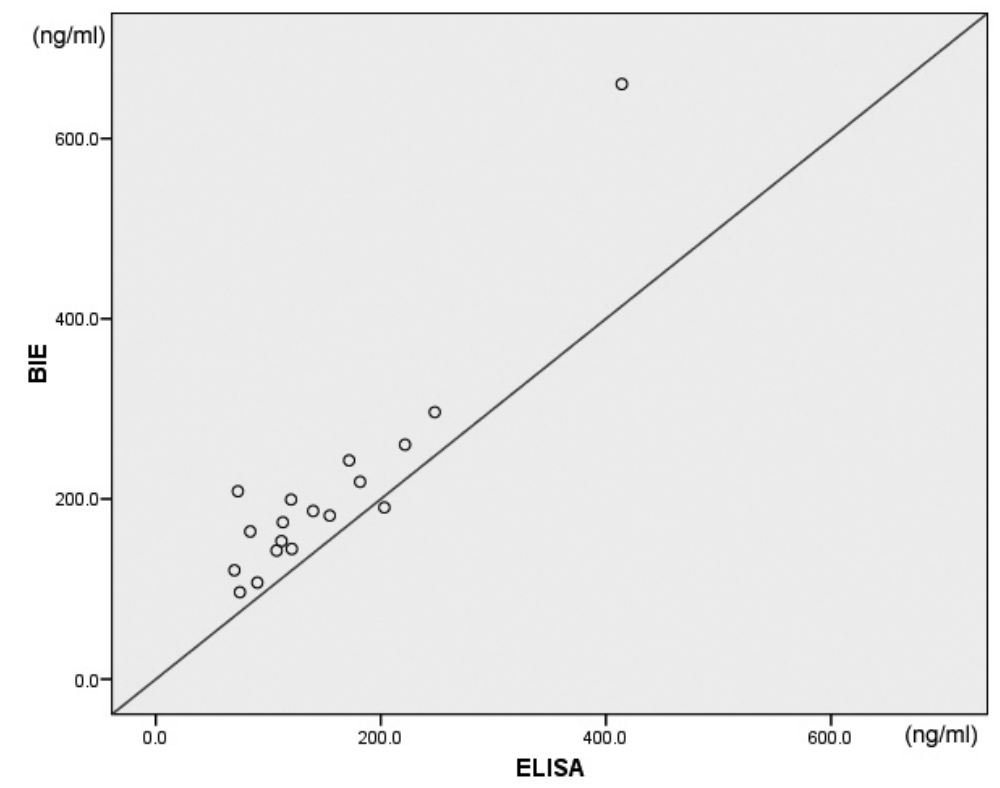

Figure 4 Comparison between BIE and ELISA for CD146 detection of 18 patient sera. The detection results of 18 patient sera measured by BIE and ELISA are plotted in the same figure, and then are performed by the correlation analysis. Pearson correlation coefficient is 0.923 which means the results of two methods are significant at the level of 0.01 .

\section{Conclusions}

Protein G is applied to bind anti-CD146 antibody and form CD146 sensing layer. It combines the process of purification of antibody in ascites sample and high oriented immobilization together.

CD146 is chosen to be a model to develop BIE as protocol tumor markers detection. CD146 is recognized and captured on its sensing surface and the detection sensitivity is beyond the order of $\mathrm{ng} / \mathrm{ml}$ grade. The results of serum sample are highly in agreement to ELISA which is a traditional biological method widely used in many aspects of protein-protein interaction research and clinical diagnosis.

\section{ACKNOWLEDGEMENT}

The author gratefully acknowledges supports from National Basic Research Program of China 2009CB320300, National High Technology Research and Development Program of China 2008AA02Z419 and Chinese Academy of Sciences (KJCX2-YW-Mo3 and -M04).

\section{REFERENCES}

[1] Parkin, D. M., "Global cancer statistics in the year 2000," Lancet Oncol. 2(9), 533-543 (2001).

[2] Sanchez-Carbayo, M., "Antibody arrays: Technical considerations and clinical applications in cancer," Clin. Chem. 52 1651-1659 (2006). 
[3] Jin, G., Tengvall, P., Lundstrom, I., and Arwin, H., "A Bionsensor concept based on imaging ellipsometry for visualization of biomolecular interactions," Anal. Biochem. 232(1), 69-72 (1995).

[4] Jin, G., Jansson, R., and Arwin, H., "Imaging ellipsometry revisited: Developments for visualization of thin transparent layers on silicon substrates," Rev. Sci. Instrum. 67(8), 2930-2936 (1996).

[5] Wang, Z. H., Meng, Y. H., Ying, P. Q., Qi, C., and Jin, G., "A label-free protein microfluidic array for parallel immunoassays," Electrophoresis 27(20), 4078-4085 (2006).

[6] Jin, G., "Development of biosensor based on imaging ellipsometry," Phys. Status Solidi A 205(4), 810-816 (2008).

[7] Qi, C., Zhu, W., Niu, Y., Zhang, H. G., Zhu, G. Y., Meng, Y. H., Chen, S., and Jin, G., "Detection of hepatitis B virus markers using a biosensor based on imaging ellipsometry," J. Viral Hepat. 16(11), 822-832 (2009).

[8] Qi, C., Lin, Y., Feng, J., Wang, Z. H., Zhu, C. F., Meng, Y. H., Yan, X. Y., Wan, L. J., and Jin, G., "Phage M13KO7 detection with biosensor based on imaging ellipsometry and AFM microscopic confirmation," Virus Res. 140(1-2), 79-84 (2009).

[9] Duan, J. Z., Yan, X. Y., Guo, X. M., Cao, W. C., Han, W., Qi, C., Feng, J., Yang, D. L., Gao, G. X., and Jin, G., "A human SARS-CoV neutralizing antibody against epitope on S2 protein," Biochem. Bioph. Res. Co. 333(1), 186-193 (2005).

[10] Shi, I. M., "The role of CD146 (Mel-CAM) in biology and pathology," J. Pathol. 189(1), 4-11 (1999).

[11] Yan, X. Y., Lin, Y., Yang, D. L., Shen, Y., Yuan, M., Zhang, Z. Q., Li, P. Y., Xia, H. T., Li, L., Luo, D. D., Liu, Q., Mann, K., and Bader, B. L., "A novel anti-CD146 monoclonal antibody, AA98, inhibits angiogenesis and tumor growth," Blood 102(1), 184-191 (2003).

[12] Sers, C., Kirsch, K., Rothbacher, U., Riethmuller, G., and Johnson, J. P., "GENOMIC ORGANIZATION OF THE MELANOMA-ASSOCIATED GLYCOPROTEIN MUC18 IMPLICATIONS FOR THE EVOLUTION OF THE IMMUNOGLOBULIN DOMAINS," P. Natl. Acad. Sci. USA 90(18), 8514-8518 (1993).

[13] Satyamoorthy, K., Muyrers, J., Meier, F., Patel, D., and Herlyn, M., "Mel-CAM-specific genetic suppressor elements inhibit melanoma growth and invasion through loss of gap junctional communication," Oncogene 20(34), 4676-4684 (2001).

[14] Wang, Z. H. and Jin, G., "A label-free multisensing immunosensor based on imaging ellipsometry," Anal. Chem. 75(22), 6119-6123 (2003).

[15] Chen, Y. Y., Meng, Y. H., and Jin, G., "Optimization of off-null ellipsometry for air/solid interfaces," Appl. Optics 46(35), 8475-8481 (2007).

[16] Wang, Z. H. and Jin, G., "Covalent immobilization of proteins for the biosensor based on imaging ellipsometry," J. Immunol. Methods 285(2), 237-243 (2004).

[17] Qi, C., Tian, X. S., Chen, S., Yan, J. H., Cao, Z., Tian, K. G., Gao, G. F., and Jin, G., "Detection of avian influenza virus subtype H5 using a biosensor based on imaging ellipsometry," Biosens.

Bioelectron. 25(6), 1530-1534 (2010). 